\title{
Comparison of Burn Depth at Different Temperatures on Ex Vivo Human Skin with Standardized Model and Comparison of the Results with Rat Contact Burn Model
}

\author{
(1) Mehmet Surhan Arda1, (1) Nilsun Kuas1, (1) Erdem Söztutar2, (1) Atacan Emre Koçman3, \\ (1) Hüseyin Illhan1 \\ 1Eskişehir Osmangazi University Faculty of Medicine, Department of Pediatric Surgery, Eskişehir, Turkey \\ ${ }^{2}$ Yeditepe University Faculty of Medicine, Department of Anatomy, İstanbul, Turkey \\ ${ }^{3}$ Eskişehir Osmangazi University Faculty of Medicine, Department of Plastic and Reconstructive Surgery, Eskişehir, Turkey
}

\begin{abstract}
Aim: Burns are still an important mortality and morbidity problem worldwide. Clinical studies are limited, owing to ethical concerns and an inability to achieve standardization. Therefore, studies are concentrated on experimental models. However, there are still a lot of questions that await resolution. Additionally, the relevance of animal models on human skin (HS) is unknown. From this point of view, this study aims to evaluate the depth of burn on ex vivo HS and to compare the HS results with those of rats.

Materials and Methods: Skins of patients, after obtaining informed consent, that underwent full thickness healthy skin excision (abdominoplasty), except for experimental purposes, have been included. A total of three different temperatures $\left(60,80\right.$ and $\left.100{ }^{\circ} \mathrm{C}\right)$ using two different weight forces $\left(0.88 \mathrm{~kg} / \mathrm{cm}^{2}\right.$ for high and $0.21 \mathrm{~kg} / \mathrm{cm}^{2}$ for low $)$ using standardized apparatus facilitated the formation of study groups. In all groups, healthy dermisepidermis burn depth was compared.

Results: No difference was detected between healthy HS depths from the various samples taken from different donors that were to be tested. The lowest result $\left(10.5 \pm 0.7 \%\right.$ burn depth ) was seen in the $60^{\circ} \mathrm{C}$ low weight force group and the highest was seen in the $100^{\circ} \mathrm{C}$ high weight force group (92.0 \pm 2.7$)$. As for the $80^{\circ} \mathrm{C}$ high pressure group vs the $100^{\circ} \mathrm{C}$ low pressure groups, a significant difference was noted.

Conclusion: Ex vivo HS can be used as an experimental burn model. It has been shown that standardized depth of burn can be achieved using standardized apparatus. However, the different depth of burn indicates that control of parameters (pressure, time, temperature) is mandatory.

Keywords: Burn, ex vivo, human skin, rat
\end{abstract}

\section{Introduction}

Burns are still a frequent trauma worldwide. According to the American Burn Association statistics, between 20062015, 205.033 individuals have suffered from burn trauma and $3.3 \%$ of them lost their lives $(1,2)$. Since the mid- $20^{\text {th }}$ century, owing to numerous experimental or clinical studies, treatment modalities have been improved. However, due to ethics and standardization problems in clinical studies, experimental studies have been mostly preferred for physiopathology and healing procedures (3-9). Therefore, various scalding and contact burn models have been defined 
(3,10-14). However, infeasible real-time contact temperatures and applied weight force (WF) measurements were the weak points of these models $(3,14)$. For this reason, in 2016 we designed a standardized contact burn model in which the real-time contact temperature and pressure could be controlled (15). A standardized second degree burn can be achieved in rats using custom designed apparatus (15). However, although the created wounds were second degree, the burn percentages were significantly different from each other. Moreover, an experimental model on human skin (HS) that defines the degree of burn has not been put forward before. With this goal in mind, this study aims to answer the following questions;

- What will be the degree of burn depth under the standardized experimental burn model?

- What are the responses and nuances of rat and HS in a standardized burn model?

\section{Materials and Methods}

This study has been conducted following approval by the human Ethics Committee (approval number: 20/02/2017$80558721 / 71)$ of Eskişehir Osmangazi University. HSs, which were assessed as waste material, were obtained from the discarded tissue of patients undergoing abdominoplasty. Before surgery informed consent was obtained from all individuals. Following excision in the operating theatre, skins were wrapped in the fresh frozen plasma (FFP) soaked gauze; transported in a vacuum bottle at $+4{ }^{\circ} \mathrm{C}$ and kept at $+4{ }^{\circ} \mathrm{C}$ until the end of procedure. All experimental steps (burns and biopsy) were performed on the same day (0 day following excision). Custom designed apparatus was used for the ex vivo HS model (Figure 1). Three temperature groups $(60,80$ and $100{ }^{\circ} \mathrm{C}$ ) and two WF groups were designed, while the elapsed time was set at $10 \mathrm{sec}$. in all groups. The burns were created using two levels of pressure upon the skin sample, with light contact being applied for the first group, defined as the Lowe WF group (LWFG), and 1.000 gr of WF being applied for the second group, defined as the (HWFG). However, real time WF force was measured in all burns due to a spring-loaded design of apparatus. Healthy normal skins of all individuals were used for a control group to measure dermis and epidermis thicknesses (Figure 2).

Group 0. Healthy HS (control)

Group 1. $60^{\circ} \mathrm{C}$ LWFG (G60LWFG)

Group 2. $60^{\circ} \mathrm{C}$ HWFG (G60HWFG)

Group $3.80^{\circ} \mathrm{C}$ LWFG (G80LWFG)

Group $4.80^{\circ} \mathrm{C} \mathrm{HWFG} \mathrm{(G80HWFG)}$

Group 5. $100^{\circ} \mathrm{C}$ LWFG (G100LWFG)

Group 6. $100^{\circ} \mathrm{C} \mathrm{HWFG} \mathrm{(G100HWFG)}$

In the laboratory, the excised skin samples were cut into strips of $10 \times 5 \mathrm{~cm}$ in length and width, and also defatted under the dermal component. These standardized tissue pieces were fixed onto a flat platform to get a perpendicular angle between the burning bar and skin for accurate measuring of WF (Figure 1) with an electronic scale. A $10 \mathrm{~mm}$ diameter cylindrical burning bar (that has $0.78 \mathrm{~cm}^{2}$ surface area) was used and at least 7 burns were created in all groups (Figure 3). One hour after the procedure, the burns were totally excised and specimens were fixed with formaldehyde.

\section{Statistical Analysis}

Slices were stained with hematoxylin eosin and examined by a blinded anatomist under light microcopy (Nikon) (Figure 4). Photographs of the burns were taken. Skin thicknesses (dermis, epidermis) and burn depths were measured from three different lines (Figure 4). Mean values and burn ratios (burned/healthy skin) were calculated for each wound using the Microsoft Excel program. Graphpad Prism 7 software was used for statistical analysis. The normality distribution of the data was assessed by the Shapiro-Wilk test. Groups were compared using a Two-Way ANOVA test with a post hoc test of Tukey's multiple comparisons. P values less than 0.05 were considered as statistically significant.

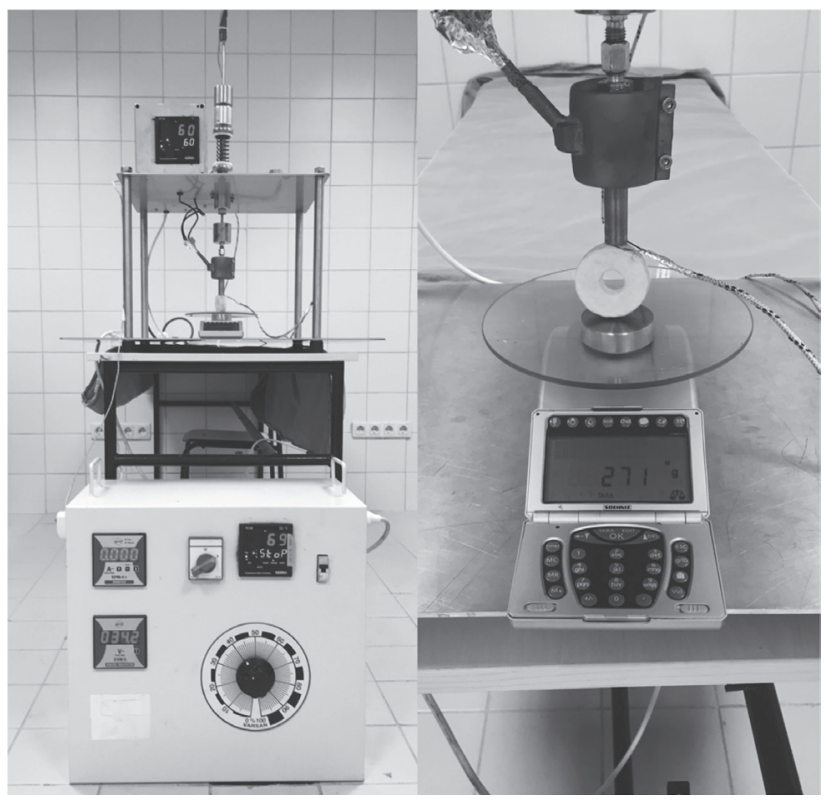

Figure 1. Custom designed apparatus

\begin{tabular}{|l|l|}
\hline \multicolumn{2}{|l|}{ Table I. Percentages of burn depth in all groups } \\
\hline Groups $\left({ }^{\circ}\right)$ & Burn depth $(\%)$ \\
\hline 60 LWFG & $10.5 \pm 0.7$ \\
\hline 60 HWFG & $25.8 \pm 2.4$ \\
\hline 80 LWFG & $52.9 \pm 2.6$ \\
\hline 80 HWFG & $71.1 \pm 2.1$ \\
\hline 100 LWFG & $66.7 \pm 2.1$ \\
\hline 100 HWFG & $92.0 \pm 2.7$ \\
\hline
\end{tabular}

LWFG: Low weight force group, HWFG: High weight force group 


\section{Results}

The discarded healthy skin of 4 patients were used. No difference was detected in the ex vivo healthy HS (dermis and epidermis) thickness of the patients ( $p>0.05)$. A mean of

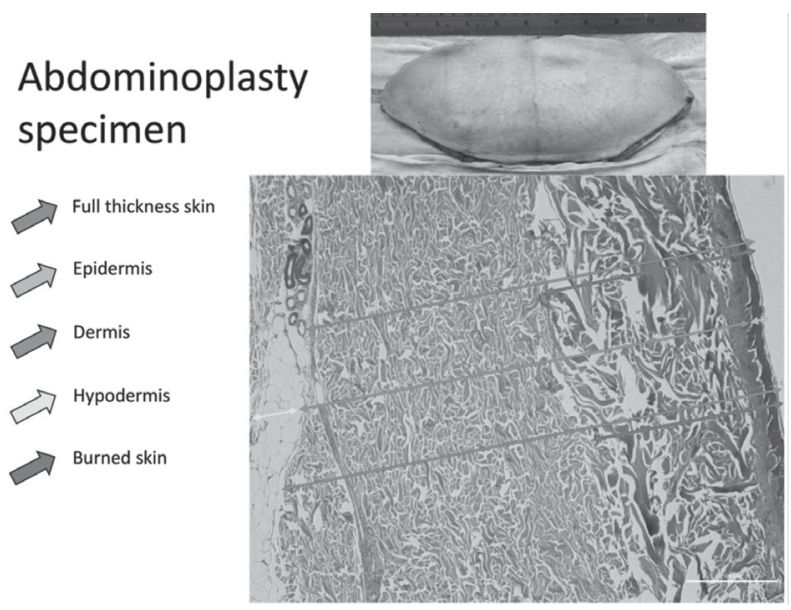

Figure 2. Skin material and histological measurements (scale=500 $\mu \mathrm{m}$ )

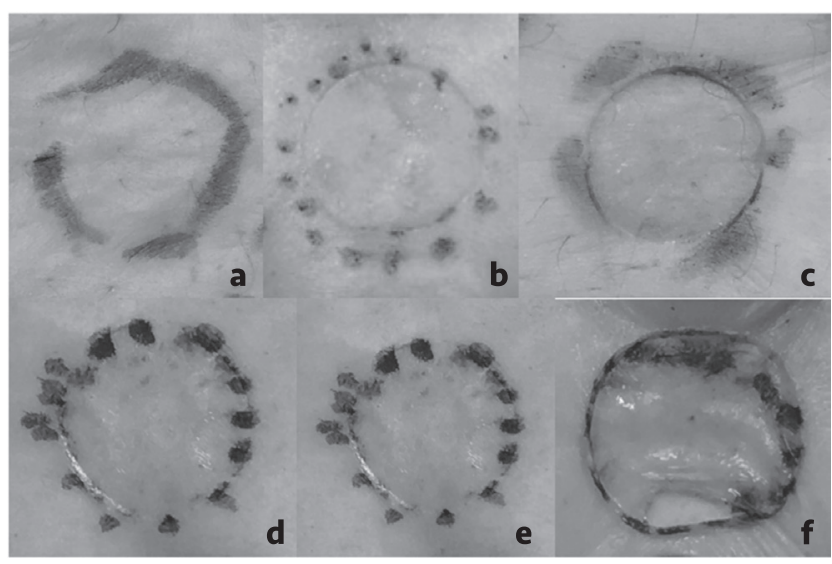

Figure 3. Macroscopic photographs of burns a) $60{ }^{\circ} \mathrm{C}$, low weight force group, b) $60^{\circ} \mathrm{C}$, high weight force group, c) $80^{\circ} \mathrm{C}$, low weight force group, d) $80^{\circ} \mathrm{C}$, high weight force group, e) $100^{\circ} \mathrm{C}$, low weight force group, f) 100 ${ }^{\circ} \mathrm{C}$, high weight force group
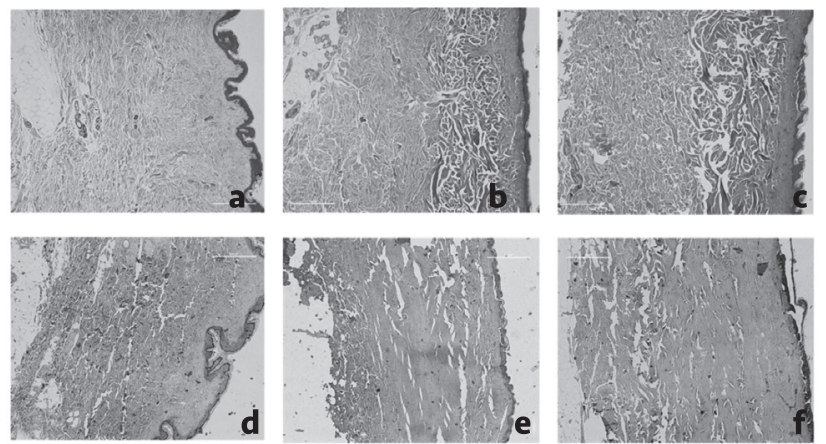

Figure 4. Representative hematoxylin\&eosin stained sections of groups. Histological appearance of a) $60^{\circ} \mathrm{C}$ low weight force group, b) $80^{\circ} \mathrm{C}$ low weight force group, c) $100{ }^{\circ} \mathrm{C}$ low weight force group, d) $60^{\circ} \mathrm{C}$ high weight force group, e) $80^{\circ} \mathrm{C}$ high weight force group, f) $100{ }^{\circ} \mathrm{C}$ high weight force group, scale bar shows 100 microns
$0.21 \mathrm{~kg} / \mathrm{cm}^{2}$ WF was applied in the LWFG and $0.88 \mathrm{~kg} / \mathrm{cm}^{2}$ in HWFG. In addition, neither in the LWFG nor in the HWFG was any difference detected ( $p>0.05$ ). The percentage of burns is given in Table I. Comparing the G80HWFG vs G100LWFG groups, highly significant different depth of burns was noted between them $(p<0.001)$. Furthermore, on ex vivo HS, in case of LWF force at $60{ }^{\circ} \mathrm{C}$, first degree burns could be created; superficial second-degree burns could be achieved if HWF is applied at the same temperature. Borderline superficial/deep second-degree burns were detected in the G80LWFG. Deep second-degree burns were confirmed in both the G80HWFG and G100LWFG groups. Third degree burns were ascertained in the G100HWFG group (Figure 3, 4).

\section{Discussion}

Burns are the most frequent trauma with an incidence of 1.1/100.000 worldwide (2). According to trauma statistics, burns are the underlying reason for approximately $5 \%$ of the patients who lose their lives due to trauma worldwide $(1,2)$. Therefore, studies concerning burns have been going on to evaluate prevention, physiopathology and treatment modalities. Cetin et al. (16) compared the survival of ex vivo HS in FFP soaked gauze and saline. And found that HS lives on in FFP for approximately thirty days. Therefore, all study procedures were performed in day 0 following excision as it is believed that results of the study would best simulate a living HS contact burn wound. Thus has not been reported on previously. Consequently, ex vivo HS and a custom designed standardized contact burn model have been used to depict the depth of burn on HS in a controlled manner. It was hoped that this study could provide a basis for a new experimental model on HS. What's more, this study could be helpful in understanding the correlation between temperature and WF on HS.

Herein, this study has shown that different statistically significant depths of burn, from $10.5 \%$ to $92 \%$ under a strict control of variables (time, temperature and WF), on ex vivo HS. In comparison to the animal model, more superficial burn depths have been noted on ex vivo HS, although there was no difference between the steps of experimental model (15). And it was realized that for Groups 1 and 6, variable depths of second degree burns were created as in the animal model. Moreover, they all have significantly different percentages of burn wounds from each other such as superficial, borderline deep/superficial and deep second degree. This might be due to different skin thicknesses or might suggest that HS is much more resistant than the skin of the rat. Finally, we believe that such different percentages of burns might cause variable inflammatory responses and that might affect the healing capacity as well. Hence, this might play an important role in inflammatory and/or healing procedures that should be evaluated. 


\section{Study Limitations}

The ex vivo nature of the study-that is without a blood supply-is its limitation. Although the skin is alive, it is impossible to account for immune reactions and put forward treatment studies. This model could be extended to become the basis for cell culture studies.

\section{Conclusion}

Due to custom designed apparatus, standard depth of burn on ex vivo HS could be investigated. The percentage of burn depth changes according to accurately controlled variables (time, WF and temperature) during the contact burn ex vivo HS has been presented. As a result, variables should be strictly under control. Especially for experimental healing models, for standardization of burns, percentage of burns might be a better indicator for classification.

\section{Acknowledgement}

Authors would like to thank to Mr. Ilthan Şeşan (retired technician) for his contribution in designing the burn apparatus.

\section{Ethics}

Ethics Committee Approval: The study was approved by the Eskişehir Osmangazi University Local Ethics Committee (approval number: 20/02/2017-80558721/71).

Informed Consent: Consent form was filled out by all participants.

Peer-review: Externally peer-reviewed.

\section{Authorship Contributions}

Concep: M.S.A., A.E.K., Design: M.S.A., A.E.K., Supervision: M.S.A., A.E.K., H.I.., Fundings: M.S.A., A.E.K., N.K., Materials: M.S.A., A.E.K., N.K., Data collection and/or Processing: M.S.A., A.E.K., N.K., E.S., Analysis and/or Interpretation: M.S.A., A.E.K., N.K., E.S., H.I., Literature Search: M.S.A., A.E.K., N.K., Writing: M.S.A., A.E.K., N.K., E.S., H.I., Critical review: M.S.A., A.E.K., N.K., E.S., H.i.

Conflict of Interest: No conflict of interest was declared by the authors.

Financial Disclosure: The authors declared that this study was funded by Eskişehir Osmangazi University Scientific Research Foundation.

\section{References}

1. American Burn Association (ABA). Burn Incidence and Treatment in the United States: 2012, http://alt.ameriburn. org/2012NBRAnnualReport.pdf

2. American Burn Association (ABA). Burn Incidence and Treatment in the United States: 2016, http://alt.ameriburn. org/2016ABANBR_FINAL_42816.pdf

3. Abdullahi $A$, Amini-Nik S, Jeschke MG. Animal models in burn research. Cell Mol Life Sci 2014;71:3241-55.

4. Campelo AP, Campelo MW, Britto GA, Ayala AP, Guimaraes SB, Vasconcelos PR. An optimized animal model for partial and total skin thickness burns studies. Acta Cir Bras 2011;26(Suppl 1):38-42.

5. Kim JY, Dunham DM, Supp DM, Sen CK, Powell HM. Novel burn device for rapid, reproducible burn wound generation. Burns 2016;42:384-91.

6. Sakamoto M, Morimoto N, Ogino S, et al. Preparation of PartialThickness Burn Wounds in Rodents Using a New Experimental Burning Device. Ann Plast Surg 2016;76:652-8.

7. Singer A), Taira BR, Anderson R, McClain SA, Rosenberg L. Does pressure matter in creating burns in a porcine model? I Burn Care Res 2010;31:646-51.

8. Venter NG, Monte-Alto-Costa A, Marques RG. A new model for the standardization of experimental burn wounds. Burns 2015:41:542-7.

9. Wang XQ, Kible RM. A review on porcine burn and scar models and their relevance to human. Wound Practice and Research 2010:18.

10. Sheu SY, Wang $\mathrm{WL}, \mathrm{Fu} Y \mathrm{YT}$, et al. The pig as an experimental model for mid-dermal burns research. Burns 2014;40:1679-88.

11. Regas FC, Ehrlich HP. Elucidating the vascular response to burns with a new rat model. J Trauma 1992;32:557-63.

12. Meyer TN, Silva ALd. A standard burn model using rats. Acta Cirurgica Brasileira 1999:14.

13. Till CO, Beauchamp C, Menapace D, et al. Oxygen radical dependent lung damage following thermal injury of rat skin. I Trauma 1983;23:269-77.

14. Sevgi M, Toklu A, Vecchio D, Hamblin MR. Topical antimicrobials for burn infections - an update. Recent Pat Antiinfect Drug Discov 2013;8:161-97.

15. Arda MS, Kocman AE, Söztutar E, Baksan B, Çetin C. A new apparatus for standardization of experimental burn models. Burns 2017:43:1322-9.

16. Cetin C, Köse AA, Aral E, et al. The effects of saline and plasma on skin graft keratinocyte viability. Br J Plast Surg 2000;53:418-9. 